\title{
Salivary cytokine levels of oral erosive lichen planus patients compared with the healthy subjects
}

\author{
Authors \\ Dr Aastha Chawla ${ }^{1}$, Dr R.D. Mehta ${ }^{2}$, Dr B.C. Ghiya ${ }^{3}$, Dr Prasoon Soni ${ }^{4}$ \\ ${ }^{1}$ Dept. of Skin \& V.D. Sardar Patel Medical College, Bikaner \\ ${ }^{2}$ Senior Professor and head, Dept. of Skin \& V.D. Sardar Patel Medical College, Bikaner \\ ${ }^{3}$ Associate Professor, Dept. of Skin \& V.D. Sardar Patel Medical College, Bikaner \\ ${ }^{4}$ Assistant Professor, Dept. of Skin \& V.D. Sardar Patel Medical College, Bikaner
}

\section{Introduction}

Lichen planus is a chronic inflammatory disease of the skin which may involve the mucosa ${ }^{1}$. Oral lichen planus is an immune-mediated disorder and an interface stomatitis characterized by $\mathrm{T}$-cell destruction $^{2}$ of the basal cells of the epithelium, mediated by Th1 cytokines. T cells release proinflammatory cytokines, interleukins (IL) namely IL-6 and IL-8. The serum and saliva levels of the interleukins serve as a reliable indicator to assess the response to treatment on a molecular basis.

TNF- $\alpha$ enhances the activation of nuclear factor$\kappa \mathrm{B}$ in the subepithelial $\mathrm{T}$ cells and as a consequence leads to the increased expression of other proinflammatory cytokines ${ }^{3,4}$ including IL6.IL-6 has been shown to be associated with multidrug resistance protein (MRP) expression by the keratinocytes ${ }^{9}$. These complex factors together have also been demonstrated to play a role in the pathogenesis of oral squamous cell carcinoma.

Cytokines are mediators that play a central role in both innate and adaptive immunity. Aberrant production of cytokines is central to the causation of immune deficiency, allergy or autoimmunity, which are involved in the pathogenesis of various immunemediated inflammatory diseases. Although a great progression has been achieved on the research of cytokines in OLP pathogenesis, there are still many unexplained aspects in this field. Cytokines do not work individually but in the complex immunomodulation pathways. It is very likely that disturbance in cytokine networks determine the progression of OLP. However, most published data are limited to the expressions of single or several cytokines in OLP, thus are not enough to explain the complexities of the cytokine network in the pathogenesis. In addition, knowledge about the intrinsic mechanisms by which the cytokine networks contribute to the chronic inflammatory environment of OLP is still lacking. Further investigation on the aberration of the cytokine network and their regulatory roles in the onset and development of OLP will deepen our understanding of the pathogenesis and lead to the identification of novel and better therapeutic approaches of this disease.

\section{Aims and Objectives}

1. To quantify the cytokine concentrations in the saliva (IL-6, IL-8, IL-1 $\beta$, TNF- $\alpha$ ) of 
oral erosive lichen planus patients and compare it with healthy controls.

2. An attempt to establish the role of these cytokines in predicting the risk of conversion of oral erosive lichen planus into the malignant oral squamous cell carcinoma.

3. To establish a correlation between the duration of the disease and the salivary cytokine levels in the patients.

4. To establish a correlation between the presence of systemic or other autoimmune diseases and the cytokine levels in patients.

\section{Methods}

A total of 25 consecutive subjects, attending the outpatient department of Dermatology were recruited, based on the below mentioned inclusion and exclusion criteria in this study after obtaining written informed consent about procedures being performed in both English and vernacular language, hereinafter termed as "cases".

\section{Inclusion Criteria}

1. Aged between the 19 and 72 years old, since OLP is an unusual disease in childhood and adolescence.

2. Presentation with characteristic clinical features of OLP (Bilateral reticular striae with atrophic erosive areas).

3. Pre-treatment Biopsy from the representative area was done, for the confirmation of diagnosis with the characteristic histopathological findings of subepithelial dense lymphocytic infiltrate, basilar vacuolization with apoptosis leading to homogeneous eosinophilic civatte bodies formation.

\section{Exclusion Criteria}

Patients were excluded from the study in cases of :

1. Negative pre-treatment biopsy for any evidence of Lichen Planus.

2. History of lichenoid contact reaction due to any medication, mouth rinse, toothpaste or any other agent;

3. Any therapy for Lichen Planus or drugs associated with Lichenoid reaction within past 8 weeks;

4. Any malignant or viral involvement in mouth;

5. Pregnant or nursing women;

6. Patients not willing to participate in the study.

After recruiting these 50 subjects in total, they were administered a semi-structured questionnaire to capture their demographic details, details of existing disease and risk factors (the questionnaire is enclosed as Annexure II). Further, their salivary samples were collected, processed and levels of IL-6, IL-8, IL-1 $\beta$, and TNF- $\alpha$ were assayed.

\section{IL-6, IL-8, IL-1ß, and TNF- $\alpha$ Assay}

The salivary samples were assayed for these cytokines at MDRU, SPMC, Bikaner laboratory using the IL-6, IL-8, IL-1ß, and TNF- $\boldsymbol{\alpha}$ enzyme linked immunosorbent assay (ELISA) kit.

\section{Results}

Maximum number of cases were in the age group of $41-50$ years $(28 \%)$ followed by $31-40$ years (24\%). Least number of cases were in the age group less than 30 years (12\%). Mean age group of cases was $47.40 \pm 14.05$ years.

Maximum number of controls were in the age group of 41-50 years (28\%) and 51-60 years (28\%) followed by $31-40$ years $(20 \%)$. Least number of controls were in the age group greater than 60 years $(8 \%)$. Mean age group of controls was $45.40 \pm 10.96$ years. 
Table Distribution of cases and controls according to age group (years)

\begin{tabular}{|c|c|c|c|c|c|}
\hline \multirow{2}{*}{$\begin{array}{ll}\text { Age } & \text { Group(In } \\
\text { Years) } & \end{array}$} & \multicolumn{2}{|c|}{ Controls } & \multicolumn{2}{|c|}{ Cases } & \multirow{2}{*}{ p value } \\
\hline & Frequency & $\%$ & Frequency & $\%$ & \\
\hline Upto 30 & 4 & $16.0 \%$ & 3 & $12.0 \%$ & \multirow{6}{*}{0.873} \\
\hline $31-40$ & 5 & $20.0 \%$ & 6 & $24.0 \%$ & \\
\hline $41-50$ & 7 & $28.0 \%$ & 7 & $28.0 \%$ & \\
\hline $51-60$ & 7 & $28.0 \%$ & 5 & $20.0 \%$ & \\
\hline$>60$ & 2 & $8.0 \%$ & 4 & $16.0 \%$ & \\
\hline Total & 25 & $100 \%$ & 25 & $100 \%$ & \\
\hline
\end{tabular}

Out of total 25 cases, 17(68\%) cases were males and $8(32 \%)$ were females. Amongst controls, $13(52 \%)$ cases were males and $12(48 \%)$ were females. The male to female ratio of cases was 2.12:1. The male to female ratio of controls was 1.08:1.
Maximum number of cases reported that illness was present since 6-12 months (32\%) followed by a duration of 6 months (24\%). Least number of cases had an illness duration ranging from 3 to 5 years $(12 \%)$.

Table Distribution of cases and controlson the basis of history of autoimmune diseases

\begin{tabular}{|l|c|c|c|c|c|}
\hline \multirow{2}{*}{$\begin{array}{l}\text { History of autoimmune } \\
\text { disease }\end{array}$} & \multicolumn{2}{|c|}{ Controls } & \multicolumn{2}{c|}{ Cases } & \multirow{2}{*}{ p value } \\
\cline { 2 - 5 } & Frequency & \% & Frequency & \% & \\
\hline No & 22 & $88.0 \%$ & 21 & $84.0 \%$ & \\
\hline Type 2Diabetes Mellitus & 2 & $8.0 \%$ & 3 & $12.0 \%$ & \multirow{2}{*}{0.894} \\
\hline Thyroid dysfunction & 1 & $4.0 \%$ & 1 & $4.0 \%$ & \\
\hline Total & 25 & $100 \%$ & 25 & $100 \%$ & \\
\hline
\end{tabular}

Four $(16 \%)$ cases had a history of autoimmune disease, out of which 3(12\%) had Type 2 Diabetes Mellitus and 1(4\%) had thyroid dysfunction.Three
(12\%) controls had a history of autoimmune disease, out of which 2(8\%) had Type 2 Diabetes Mellitus and 1(4\%) had thyroid dysfunction.

Table Distribution of cases and controlson the basis of history of systemic illness

\begin{tabular}{|c|c|c|c|c|c|}
\hline \multirow{2}{*}{ History of systemic illness } & \multicolumn{2}{|c|}{ Controls } & \multicolumn{2}{|c|}{ Cases } & \multirow{2}{*}{$\begin{array}{c}\mathbf{p} \\
\text { value }\end{array}$} \\
\hline & Frequency & $\%$ & Frequency & $\%$ & \\
\hline No & 19 & $76.0 \%$ & 16 & $64.0 \%$ & \multirow{6}{*}{0.516} \\
\hline Anxiety disorder & 0 & $0.0 \%$ & 1 & $4.0 \%$ & \\
\hline Hypertension & 6 & $24.0 \%$ & 6 & $24.0 \%$ & \\
\hline $\begin{array}{l}\text { Hypertension, } \\
\text { Coronary artery disease(CAD) }\end{array}$ & 0 & $0.0 \%$ & 1 & $4.0 \%$ & \\
\hline $\begin{array}{l}\text { Hypercholesterolemia,Abnormal } \\
\text { LFT, Hyperbilirubinemia }\end{array}$ & 0 & $0.0 \%$ & 1 & $4.0 \%$ & \\
\hline Total & 25 & $100 \%$ & 25 & $100 \%$ & \\
\hline
\end{tabular}

Nine cases of OLP had an associated systemic illness. Six (24\%) patients had systemic hypertension, $1(4 \%)$ had hypertension along with coronary artery disease, 1(4\%) had hypercholesterolemia, abnormal hepatic profile, hyperbilirubinemia and 1(4\%) had anxiety disorder. Amongst control group, 6(24\%) people had sytemic illness in the form of hypertension. Most common site of oral mucosa involvement was buccal mucosa, seen in all 25 cases(100\%) followed by involvement of lip, seen in 10 cases $(40 \%)$. The least common site of oral mucosa involvement was palate, noted in 2 cases $(8 \%)$

1. For IL-6, the presence of an autoimmune disease was not associated with higher cytokine values ( $\mathrm{p}$ value $=0.976$ )

2. For IL-8, the presence of an autoimmune disease was associated with higher cytokine values ( $\mathrm{p}$ value $=0.015)$. 
3. For TNF- $\alpha$, the presence of an autoimmune disease was not associated with higher cytokine values $(\mathrm{p}$ value $=$ 0.676).

4. For IL-1 $\beta$, the presence of an autoimmune disease was not associated with higher cytokine values $(\mathrm{p}$ value $=0.680$ )

\section{Conclusion}

We conducted a study to quantify the cytokine concentrations in the saliva (IL-6, IL- 8, TNF- $\alpha$, IL-1 $\beta$ ) of oral erosive lichen planus patients and compared these with healthy controls so as to establish the role of these cytokines in predicting the risk of conversion of oral erosive lichen planus into the malignant oral squamous cell carcinoma. A total of 25 cases, and 25 controls with good oral and general health, were enrolled under this study with standard informed consent. They were administered a questionnaire to capture their demographic details, details of existing disease and risk factors (the questionnaire is enclosed as Annexure II). Further, their salivary samples were collected, processed and levels of IL-6,IL-8, TNF$\alpha$ and IL-1 $\beta$ were assayed.

In our study, maximum number of cases were in the age group of 41-50 years (28\%). Mean age group of the cases was $47.40 \pm 14.05$ years. Maximum number of controls were in the age group of 41-50 years (28\%) and 51-60 years (28\%). Mean age group of controls was $45.40 \pm$ 10.96 years.

The male to female ratio of cases was 2.12:1.The male to female ratio of controls was 1.08:1. Mean duration of illness in the 25 cases studied was $30.48 \pm 35.99$ months. There was no stastically significant association between the duration of illness and the level of cytokines.

Out of 25 cases, only 1(4\%) had a positive family history. Four $(16 \%)$ cases had a history of autoimmune disease. Three (12\%) controls had a history of autoimmune disease. Amongst the cases; presence of an autoimmune disease was not associated with higher IL-6, TNF- $\alpha$ and IL-1 $\beta$ values. However, for IL-8, the presence of an autoimmune disease was associated with higher cytokine values. Amongst the controls; presence of an autoimmune disease was not associated with higher IL-6, IL-8, TNF- $\alpha$ and IL- $1 \beta$ values.

Nine cases in the study group had an associated systemic illness. Amongst controls, 6(24\%) people had hypertension. Amongst both,the cases and controls; presence of a systemic illness was not associated with higher IL-6, IL-8, TNF- $\alpha$ and IL- $1 \beta$ values.

Most common site of oral mucosa involvement was buccal mucosa, seen in all 25 cases $(100 \%)$ followed by involvement of lip, seen in 10 cases (40\%). The least common site of involvement was palate, noted in 2 cases ( $8 \%$ ).

Cutaneous LP along with oral erosive LP was present in $4(16 \%)$ out of 25 cases.

Only $3(12 \%)$ out of 25 cases had not received any previous treatment for their condition, rest $22(88 \%)$ cases had already received treatment in the form of topical or systemic medications. For all the four cytokines, previous treatments received did not affect the cytokine values ( $p$ value $>0.05$ ).

Salivary IL-6, IL-8,TNF- $\alpha$, IL-1 $\beta$ values of patients with oral erosive LP was higher than that of controls and the association was statistically significant.

This study confirms the effect of immune cytokine in pathogenesis of oral lichen planus. The fact that these cytokines in saliva were significantly elevated in the patients may have diagnostic and prognostic utility as useful biomarkers and indicators of carcinogenic transformation from Oral erosive Lichen planus to cancer. However larger sample size may be required to prove this in a conclusive manner

\section{Bibliography}

1. Carrozzo M, Uboldi de Capei M, Dametto E, Fasano ME, Arduino P, Broccoletti R, et al. Tumor necrosis factor-alpha and interferongamma polymorphisms contribute to susceptibility to oral lichen planus. J Invest Dermatol. 2004;122(1):87-94. 
2. Lavanya N, Jayanthi P, Rao UK, Ranganathan K. Oral lichen planus: An update on pathogenesis and treatment. Journal of Oral and Maxillofacial Pathology: JOMFP. 2011;15(2):127-132.

3. Gu GM, Martin MD, Darveau RP, Truelove E, Epstein J. Oral and serum IL-6 levels in oral lichen planus patients. Oral Surg Oral Med Oral Pathol Oral Radiol Endod. 2004;98(6):673-8.

4. Sun A, Chia JS, Chang YF, Chiang CP. Serum interleukin- 6 level is a useful marker in evaluating therapeutic effects of levamisole and Chinese medicinal herbs on patients with oral lichen planus. J Oral Pathol Med. 2002;31(4):196-203. 